\title{
Turning objects on their heads: The influence of the stored axis on object individuation
}

\author{
IRINA M. HARRIS and PAUL E. DUX \\ Macquarie University, Sydney, New South Wales, Australia
}

\begin{abstract}
We used repetition blindness (RB) as a measure of object recognition and compared the pattern of $\mathrm{RB}$ obtained for objects with a well-established upright orientation (mono-oriented objects) and those without a usual upright orientation (polyoriented objects), when the critical objects were either in identical orientations or differed by $30^{\circ}, 60^{\circ}, 90^{\circ}$, or $180^{\circ}$. Overall, we found robust RB despite differences in orientation, consistent with the idea that object recognition, as indexed by RB, is largely independent of orientation. However, whereas for polyoriented objects RB was obtained in all orientation conditions, for mono-oriented objects there was no RB between upright and upside-down versions of the stimuli. These findings suggest that the usual orientation of an object-when it exists-is stored in memory and can facilitate orientation processing when the principal axis of a viewed object is aligned with the stored axis orientation. This, in turn, allows for more rapid and successful construction of distinct episodic representations of an object, thus alleviating RB.
\end{abstract}

One of the best-known and most widely accepted notions in visual neuroscience is that the brain does not process and represent visual stimuli in a unitary manner, but rather contains highly specialized modules dedicated to processing different attributes such as shape, color, spatial location, and motion. A striking example of independent processing of different visual attributes comes from a neuropsychological syndrome known as agnosia for object orientation (Harris, Harris, \& Caine, 2001; Karnath, Ferber, \& Bülthoff, 2000; Turnbull, Beschin, \& Della Sala, 1997; Turnbull, Laws, \& McCarthy, 1995). Patients with this syndrome show a dissociation between their ability to recognize objects presented in different orientations and their ability to determine the objects' orientations. Typically, their recognition accuracy is very high, even when the objects are rotated away from their usual upright orientation. In contrast, their ability to determine the orientation of an object is severely impaired. In general, these patients are very poor at judging whether an object is in its correct (e.g., upright) orientation, they have great difficulty discriminating between identical objects that differ only in orientation, and some of them make orientation errors when copying drawings of objects (e.g., Turnbull et al., 1997). Interestingly, other aspects of performance, such as their reaction times when naming rotated objects, are quite insensitive to orientation manipulations (Turnbull, Della Sala, \& Beschin, 2002), unlike normal observers whose

This study was supported by an Early Career Researcher grant from Macquarie University. I.M.H. was supported by an ARC Australian Postdoctoral Fellowship, and P.E.D. was supported by an Australian Postgraduate Award. We thank Vince Polito for assistance in testing subjects, and Charles Leek and Steve Lupker for helpful comments. Correspondence should be addressed to I. M. Harris, School of Psychology, University of Sydney, Sydney NSW 2006, Australia (e-mail: irina@ psych.usyd.edu.au). reaction times typically increase in a systematic fashion as the object is rotated farther from the upright orientation (e.g., Jolicœur, 1985). Thus, it appears that these patients' object recognition is mediated by orientation-independent representations, consistent with the idea that object identity and orientation are processed by different neural systems (Milner \& Goodale, 1995; Turnbull et al., 1997).

In spite of these patients' severe deficits in determining object orientation, certain aspects of their performance nonetheless suggest some degree of sensitivity to the correct orientation of the object. For example, one patient was better able to match the orientation of objects when given an upright model (Turnbull et al., 1995), and another patient tended to copy misoriented drawings in the upright orientation (Turnbull et al., 1997). An even clearer demonstration of preserved knowledge of upright orientation comes from two more recent cases who, despite performing poorly with misoriented objects, made no errors in judging the orientation of upright objects (Harris et al., 2001; Karnath et al., 2000). These patients were also more likely to choose upright orientation when asked to reorient pictures in order to demonstrate their canonical orientation.

Furthermore, one of these patients, E.L., was significantly more accurate in judging the orientation of upsidedown objects, compared with those rotated $90^{\circ}$ (Harris et al., 2001). He was also better at discriminating between an upright and an (identical) upside-down object than between other orientations of the object, such as the upright orientation and a $90^{\circ}$ rotation, or between an object rotated $90^{\circ}$ clockwise and the same object rotated $90^{\circ}$ counterclockwise. Thus, in addition to a preserved ability to recognize the correct upright orientation, E.L. demonstrated some facility in processing the orientation of upside-down objects. This facility is not unique to E.L. Patient K.B., reported by Karnath et al. (2000), was also more often correct in judging the orientation of inverted objects, com- 
pared with those rotated $90^{\circ}$. Similarly, when attempting to reorient wooden letters, she apparently made some errors by placing the letters at $90^{\circ}$ or mirror-reversed, but never upside-down. In the same vein, Patient N.L. tended to copy misoriented drawings in an upright orientation, but only did so for models presented $90^{\circ}$ from the upright orientation; he copied upside-down objects correctly in an upside-down orientation (see Turnbull et al., 1997, Figure 5). In addition, normal participants are more accurate at judging the orientation of briefly presented upright and upside-down objects, compared with those rotated $90^{\circ}$ (De Caro, 1998; Harris \& Dux, 2005, Experiment 3).

To explain this pattern of results, Harris et al. (2001) suggested that object representations stored in memory must contain some information about the location of the top-bottom axis of the object (i.e., its usual orientation). As a consequence, the orientation of an upright object is interpreted with ease, because in this case there is a perfect match between the top-bottom axis of the viewed object and the axis information stored in memory. Upsidedown objects also benefit to some extent, because in this case the location of the top-bottom axis is partially predicted by the stored axis information (i.e., they are both vertically aligned). In contrast, other orientations are more difficult to interpret because in those cases the top-bottom axis is no longer in the predicted location. Therefore, the principal axis of the object has to be located and compared with that of the stored representation. Patients with orientation agnosia seem unable to carry out this step.

Such an explanation might initially seem difficult to reconcile with the notion that these patients' recognition is mediated by orientation-invariant object representations. However, it is important to note that whatever sensitivity to orientation is evidenced by these patients, it is only apparent in their performance on orientation judgments. As outlined above, their recognition accuracy seems insensitive to orientation. This state of affairs implies that the orientation information coded in memory is somewhat independent of the object representation that mediates recognition. For example, the object representation stored in memory may comprise a distributed collection of separate pieces of information, such as an orientation-free shape description, information about the canonical orientation of the object (or the top-bottom axis), as well as other semantic attributes. Therefore, an orientation-free object representation may still be sufficient to locate a match in memory and thus enable recognition, even in the absence of information about the object's orientation (Corballis, 1988).

Recently, we have obtained evidence supporting this idea from a completely different source-namely, normal participants' performance on a repetition blindness task involving rotated objects (Harris \& Dux, 2005). Repetition blindness (RB) refers to a reduced capacity to report both occurrences of a repeated item in a rapid serial visual presentation stream, when they occur within approximately $400-500 \mathrm{msec}$ of each other (Kanwisher, 1987). This failure to report the repeated item is not due to masking or limitations in short-term memory, because a different item presented at the same temporal position is usually detected and reported successfully. RB is generally thought to reflect a failure to assign separate episodic tokens to a repeatedly activated type (Kanwisher, 1987). In this context, a distinction is made between types, which are long-term representations stored in memory, and tokens, which are episodic representations of particular instances of a visual type (Kahneman \& Treisman, 1984). Thus, one can think of RB as an example of implicit recognition, because some form of recognition must take place in order to give rise to the repetition effect, even though the repeated stimulus is not consciously perceived as a separate visual event or episode.

In our previous study, subjects had to report three pictures of objects presented in rapid succession, in various picture-plane orientations $\left(0^{\circ}, 30^{\circ}, 60^{\circ}, 90^{\circ}, 180^{\circ}\right)$. The first and third pictures were the critical items and could be either identical objects (repeated condition) or different objects (nonrepeated condition); moreover, the critical items were either in the same orientation (both upright) or differed in orientation. We obtained significant RB across all orientation differences tested, which suggests that the representations that mediate the RB effect (and, implicitly, recognition) are orientation invariant (Harris \& Dux, 2005). These findings extend those of a previous study that found similar orientation-invariant RB for small orientation differences of $30^{\circ}$ (Kanwisher, Yin, \& Wojciulik, 1999).

Interestingly, we also found that, under some conditions, RB was substantially diminished, or even completely absent, when the repeated objects were upright and upside-down (i.e., $0^{\circ}$ vs. $180^{\circ}$ orientations). This finding is reminiscent of the results obtained from the orientation agnosics described earlier, which indicate that the upright and upside-down orientations are easier to determine and discriminate. We reasoned that, if subjects can readily interpret the objects' orientation in this case, they can use that information as an individuating feature to help them encode distinct episodic representations of that object and consequently report both of them. In contrast, other rotated orientations are more difficult to determine, making it difficult for subjects to extract sufficiently reliable orientation information from the briefly displayed pictures to be confident that they represent different tokens of the same identity type.

As outlined earlier, Harris et al. (2001) hypothesized that this facility with upright and upside-down objects is due to the fact that, in these cases, the top-bottom axis of the object is aligned with the axis information stored in memory (i.e., the object's usual orientation). One way to test this hypothesis is to examine performance with a category of objects that do not have a typical, or predominant, orientation - things like pens, keys, or hammers. In the present study, we contrasted the pattern of RB obtained across different orientations for objects with a well-established canonical upright orientation (monooriented objects - e.g., cup, kettle, shoe) and objects without a canonical upright orientation (polyoriented objectse.g., fork, scissors, whistle). On the basis of our previous 
results, we expected to see robust $\mathrm{RB}$ for all orientation differences, except $180^{\circ}$, for mono-oriented objects. In contrast, for polyoriented objects, we expected to see a uniform pattern of RB for all orientation conditions, including differences of $180^{\circ}$, because in this case the $0^{\circ}$ and $180^{\circ}$ orientations of these items would be arbitrary and not related to a default orientation stored in memory (Leek, 1998).

\section{METHOD}

\section{Subjects}

Thirty-one psychology students (20 females and 11 males), $17-59$ years of age $($ mean $=23)$, participated in the experiment in exchange for either course credit or a $\$ 10$ payment.

\section{Apparatus}

The stimuli were presented on a Dell Flat Trinitron monitor with a $120-\mathrm{Hz}$ vertical refresh rate controlled by a Dell computer. The experiment was constructed and run with DMDX software (Forster \& Forster, 2003).

\section{Stimuli}

The stimuli were 48 line drawings from the Snodgrass and Vanderwart (1980) corpus. They were presented as black line drawings on a white background, subtending a visual angle of approximately $12^{\circ}$ at a viewing distance of $45 \mathrm{~cm}$. Three masks were created using random geometrical shapes of line thickness and density similar to those of the pictures.

Thirty-two of the pictures served as critical items. Half of these were objects with a well-established canonical upright orientation (baby carriage, basket, bird, candle, cup, kettle, mushroom, sailing boat, shoe, snowman, stove, telephone, trash can, tree, truck, wineglass), whereas the other half were objects that are habitually encountered in many different orientations (belt, brush, clothes peg, corn, fork, French horn, guitar, lock, mitten, nail file, onion, pliers, saw, scissors, watch, whistle). The two sets of objects were equated in terms of mean visual complexity $[2.43 \mathrm{vs} .2 .83, t(15)=.10]$ and familiarity ratings [3.66 vs. $3.42, t(15)=.43$ ], on the basis of the Snodgrass and Vanderwart (1980) norms.

The remaining 16 objects served as intervening items presented between the two critical items. These were all mono-oriented objects (bed, bus, chicken, cow, dress, dresser, frog, frying pan, giraffe, gun, jacket, monkey, pitcher, rhinoceros, stool, windmill).

\section{Experimental Design}

Three independent variables were manipulated in a $2 \times 2 \times 5$ within-subjects design (see Figure 1). The first variable was object type: mono-oriented or polyoriented. The second variable was the relationship between the two critical items: repeated (same object) or nonrepeated (different object, of the same object type). The third factor was the orientation difference between the critical items: $0^{\circ}$, $30^{\circ}, 60^{\circ}, 90^{\circ}$, or $180^{\circ}$. For mono-oriented objects, the degree of misorientation was defined relative to the usual upright orientation $\left(0^{\circ}\right)$ of the object, whereas for the polyoriented objects, the degree of misorientation was defined in relation to the stimulus orientation depicted in the Snodgrass and Vanderwart (1980) set. The first critical item $(\mathrm{C} 1)$ always appeared upright or in its nominal $0^{\circ}$ orientation, whereas the orientation of the second critical item (C2) was varied. The intervening items were always upright.

\section{Procedure}

The experiment was conducted under normal illumination conditions and lasted approximately $1 \mathrm{~h}$. The subjects were seated $\sim 45 \mathrm{~cm}$ in front of the computer monitor and gave their responses verbally; these were coded by the experimenter. Before the experiment, the subjects completed a familiarization phase in which they saw all the pictures (in their upright, or nominal $0^{\circ}$, orientation) and named them. Any naming errors were corrected at this stage.

The experiment was self-paced, and the subjects initiated each trial by pressing the space bar. Each trial consisted of three pattern masks, followed by the three pictures $(\mathrm{C} 1$, intervening item, $\mathrm{C} 2)$, followed by the same three pattern masks in reverse order. Each of these frames was presented for $100 \mathrm{msec}$ with no interstimulus in-

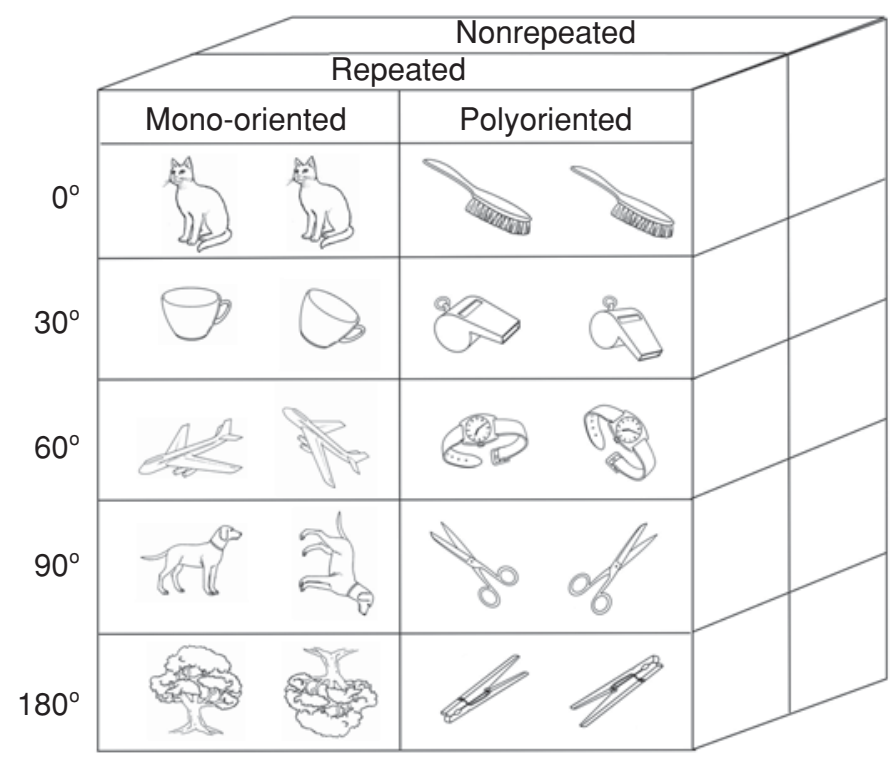

Figure 1. Graphical representation of the experimental design. Three factors were manipulated: (1) The type of object (mono-oriented or polyoriented), (2) the relationship between the critical items (same or different object), and (3) the orientation of the rotated item. The critical items depicted here were separated by an intervening object (not shown). 

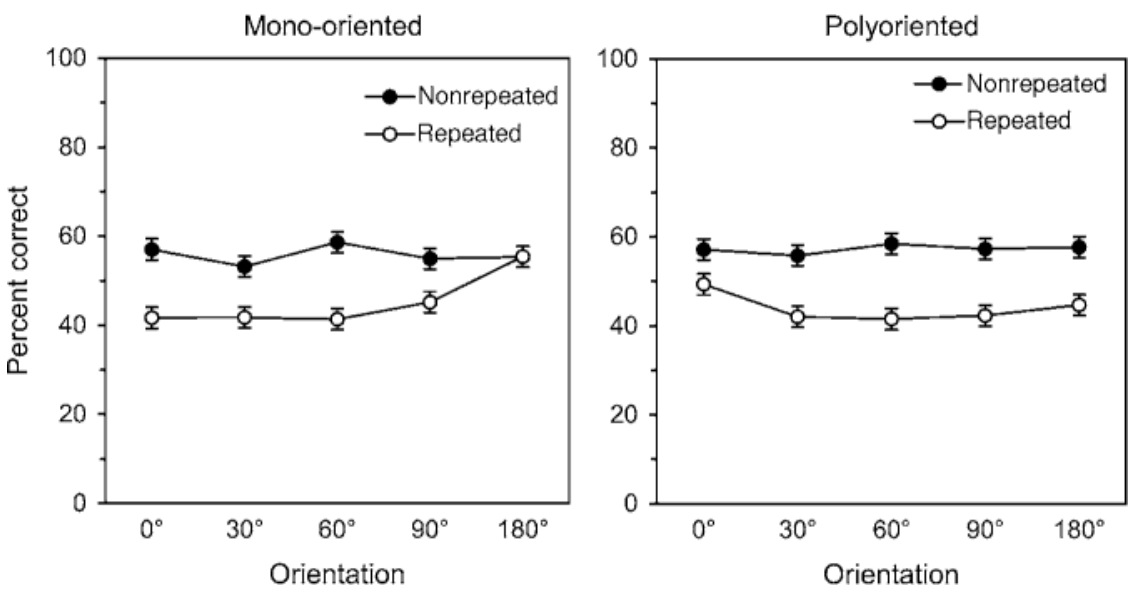

Figure 2. Mean percent correct recall of both critical items for repeated and nonrepeated trials, as a function of the object's orientation, shown separately for mono-oriented objects and polyoriented objects. Error bars represent within-subjects standard error of the mean (Loftus \& Masson, 1994).

terval. At the end of the trial, a message appeared on the screen, prompting the subjects to recall all the pictures seen. They were told that sometimes a picture was repeated and, if that were the case, they should name it twice. In addition to the 320 experimental trials $(2 \times 2 \times 5 \times 16), 32$ filler trials containing only two pictures (one critical item and one intervening item) were included, in order to discourage the subjects from guessing about the presence of an undetected repeated picture. An extra mask was presented on these trials, sometimes before the pictures and sometimes after them, in order to equate the number of frames. The trials were randomly intermixed. Before the start of the experiment, the subjects received 32 practice trials at increasingly fast presentation rates ranging from $500 \mathrm{msec}$ per picture ( 8 trials), to $150 \mathrm{msec}$ per picture ( 8 trials), to $100 \mathrm{msec}$ per picture (16 trials).

\section{RESULTS}

The data from 4 subjects were excluded from analysis because of near-ceiling performance (more than $80 \%$ correct overall). The exposure duration was clearly too long for these subjects to show any effects of the experimental manipulation.

Naming errors constituted very few of the errors (1\% or less); mostly, subjects just failed to report all three items. Trials on which neither $\mathrm{C} 1$ nor $\mathrm{C} 2$ were reported were discarded. This means that, for repeated objects, we only included trials for which we could be sure that the object representation of interest had been activated. For symmetry, the same criterion was applied to nonrepeated items. We opted for this method, rather than the more stringent method of counting only trials on which $\mathrm{C} 1$ was reported, because it is often impossible to distinguish which of the repeated items is reported in these tasks. The data were then scored in terms of percent of trials in which both $\mathrm{C} 1$ and $\mathrm{C} 2$ were correctly recalled and are shown in Figure 2, plotted separately for monooriented objects and polyoriented objects.

As can be seen, overall levels of accuracy were similar for the two object types. Performance was generally worse on repeated trials, which is the hallmark of $\mathrm{RB}$, al- though RB was alleviated in the case of mono-oriented objects when they differed by $180^{\circ}$. This was not the case for polyoriented objects, where robust RB was observed in all orientation conditions.

A $2 \times 2 \times 5$ repeated measures ANOVA confirmed this pattern of results. There was no main effect of object type $[F(1,26)=0.013, p=.91]$, but there was a significant main effect of repetition $[F(1,26)=9.16, p<.01]$, and a significant main effect of orientation $[F(4,104)=$ $2.93, p<.05]$. Object type did not interact with repetition $[F(1,26)=0.77, p=.39]$, or with orientation $[F(4,104)=1.976, p=.10]$. However, there was a significant two-way interaction between repetition and orientation $[F(4,104)=2.56, p<.05]$, as well as a significant three-way interaction $[F(4,104)=2.91, p<.05]$, confirming that RB was only modulated by orientation for mono-oriented objects. Simple comparisons revealed significant differences between repeated and nonrepeated trials for orientation differences of $0^{\circ}, 30^{\circ}, 60^{\circ}$, and $90^{\circ}$ (all $F_{\mathrm{s}}>9.71$, all $\left.p \mathrm{~s}<.005\right)$, but not $180^{\circ}[F(1,104)<$ $1, p>.98]$, in the case of mono-oriented objects. For polyoriented objects, all differences between repeated and nonrepeated trials were significant $\left(F_{\mathrm{S}}>6.27, p \mathrm{~s}<\right.$ $.02)$.

\section{DISCUSSION}

In this study, we contrasted the pattern of RB obtained across changes in orientation for objects with a wellestablished canonical orientation and for those without a canonical orientation. In general, we found robust RB despite differences in orientation, although there was one notable difference between the two categories of objects. For polyoriented objects, significant RB was present in all orientation conditions, whereas for mono-oriented objects there was no RB for upright versus upside-down versions.

This difference in the pattern of RB is unlikely to be due to differences in item difficulty, given that accuracy 
levels were essentially identical for the two categories of objects. At the same time, the fact that RB was maintained for polyoriented objects that differed by $180^{\circ}$ indicates that the loss of RB for upright and upside-down objects was not due simply to the fact that a $180^{\circ}$ difference in orientation, in itself, makes the stimuli more discriminable and, therefore, easier to individuate. Thus, the difference seems more likely to arise from a qualitative difference between these two classes of objects.

A potential difference between the two object categories, as tested here, is in the orientation of their principal axis of elongation. For example, because the orientation depicted in the Snodgrass and Vanderwart (1980) corpus was chosen as the nominal $0^{\circ}$ for the polyoriented objects, for quite a few of the polyoriented objects (e.g., clothes peg, corn, fork) the orientation of the principal axis of elongation of the object was at about $60^{\circ}$ from the vertical. Therefore, when the objects differed by $180^{\circ}$ their principal axes of elongation were oriented at $60^{\circ}$ and $240^{\circ}$. In contrast, for the mono-oriented objects the $180^{\circ}$ condition involved two objects with vertically aligned principal axes. Thus, it could be argued that the loss of RB for upright and upside-down mono-oriented objects was due to the fact that it is easier to interpret the orientation of objects with a vertical axis of elongation, compared with an oblique axis. However, there are a few reasons to discount such an explanation, both in terms of the composition of the stimulus sets in this experiment and on empirical grounds.

First, quite a number of polyoriented objects used in the present experiment did not have an obvious axis of elongation (e.g., belt, French horn, onion, watch, whistle), so it does not make sense to ponder whether these objects' principal axes are vertical or oblique. At the same time, a number of the mono-oriented objects did not have a vertical axis of elongation, either (e.g., the shoe and the truck have a horizontal axis of elongation, whereas the telephone and the cup do not have an obvious axis of elongation). Thus, it seems unlikely that our results could be explained by differences in object geometry between the two categories. Second, it has been demonstrated empirically that the axis of elongation plays no special role in the recognition of rotated monooriented objects (Large, McMullen, \& Hamm, 2003). Third, Leek (1998) carried out a careful study of the representation of polyoriented objects and failed to find any evidence that they may be preferentially represented in particular orientations, including those in which the principal axis of elongation is vertical.

Further evidence that differences in the orientation of the axis of elongation were not responsible for our results comes from additional analyses of the present data. We divided both the mono-oriented and the polyoriented objects into two groups: objects with a clear axis of elongation (e.g., candle, nail file, scissors, tree) and objects that did not have such an obvious axis (e.g., basket, French horn, telephone, whistle). These four groups of objects were compared in terms of performance accuracy on the repeated trials in the $180^{\circ}$ condition (this analysis could not be carried out on nonrepeated trials because those object pairs frequently contained combinations of objects with and without an axis of elongation). Note that in the case of objects with a principal axis of elongation, the mono-oriented objects had predominantly vertical axes in the $0^{\circ}$ and $180^{\circ}$ orientations, whereas the polyoriented objects all had oblique axes of elongation. Therefore, if the vertical orientation of the principal axis of elongation was responsible for the loss of RB between upright and upside-down mono-oriented objects, one would expect to see an interaction between object type and presence/absence of an axis of elongation. A $2 \times 2$ ANOVA confirmed that mono-oriented objects were reported more accurately than polyoriented objects $[F(1,26)=8.13, p<.01]$, reflecting the loss of RB evident in the overall analysis. In addition, there was no effect of axis $[F(1,26)=3.12, p=.09]$ and, crucially, no interaction between object type and presence/absence of axis of elongation $[F(1,26)=0.85, p=.37]$. Therefore, the more successful individuation seen in this study for mono-oriented objects appears to be related to the upright and upside-down orientations of objects that have a wellestablished upright orientation, rather than to the orientation of the principal axis of elongation.

Interestingly, a similar pattern of performance was reported in a patient who could discriminate between upright and upside-down versions of objects with a "base" (i.e., a usual upright orientation), but was much less accurate when discriminating between objects without a base, or abstract shapes, that differed by $180^{\circ}$ in the vertical dimension (Davidoff \& Warrington, 1999). These findings are consistent with the idea that the usual orientation of an object - when it exists - is stored in memory and can facilitate the interpretation of those orientations in which the top-bottom axis of the object is aligned with the stored axis information. Although the nature of this axis information remains open to empirical investigation, Harris et al. (2001) have suggested that the stored axis information could be conceptualized as a set of predictions about the spatial configuration of the object features in an object-centered reference frame. These predictions would provide top-down information about the usual location of the principal axis of the object in retinal or image coordinates. Therefore, when objects are rotated away from their usual orientation, a mismatch occurs between the location of the axis in the image and the location expected on the basis of the information stored in memory, resulting in performance costs. These costs are reduced for upside-down objects, because in this case the object's principal axis is still in the expected location, leading to more rapid encoding of the object's orientation. The present results show that this, in turn, allows the subject to rapidly establish distinct episodic representations of upright and upside-down objects, thus eliminating RB between such objects (see also Harris \& Dux, 2005).

With the exception of this special case of upright and upside-down objects, the overall pattern of performance was one of robust RB across changes in orientation. This 
implies that the representations that mediate object recognition, as indexed by $\mathrm{RB}$, are invariant with respect to orientation. It is, of course, possible that the RB effect is mediated by semantic or phonological representations, which are orientation invariant in a rather trivial sense. Bavelier (1994) found RB between pictures and words depicting the same object, and Kanwisher et al. (1999) have demonstrated RB between different exemplars of the same basic-level object (e.g., grand piano and upright piano) and for semantically related objects, although not between objects that are only phonologically similar. Therefore, an explanation in terms of semantic RB, at least, is plausible. However, if RB occurred at a semantic level, it is hard to see why the effect would disappear for upright and upsidedown versions of objects with a canonical orientation. We have seen this loss of RB for upright versus upside-down objects in previous experiments (Harris \& Dux, 2005), so we can conclude that it is a robust finding. Thus, we would argue that the RB effect in this paradigm is more likely to occur at a presemantic than semantic or phonological level of representation, a conclusion that is also supported by findings of RB between pseudo-objects that have no semantic or phonological representation (Arnell \& Jolicœur, 1997).

In conclusion, the present results provide additional evidence for the dissociation between object recognition and the representation of object orientation. Object recognition, as indexed by $\mathrm{RB}$, was found to be largely independent of orientation, consistent with the idea that recognition in this context is mediated by orientation-free representations. At the same time, however, it is quite clear that if an object has a predominant or usual orientation, this information is also stored in memory. That said, the results of the present study, along with those obtained from patients with orientation agnosia, highlight the fact that this information is stored separately from the shape representation that mediates recognition and that its effects are dissociable from the recognition process (see also De Caro \& Reeves, 2000). Therefore, it seems to us that the best way to conceptualize the memory representation of an object is as a distributed collection of attributes, including shape and identity, usual orientation, and other semantic information, rather than as a unitary image-like representation of a particular shape in a particular orientation. Thus, activating shape and identity information, without necessarily processing the object's orientation, may be sufficient to enable at least some forms of recognition (Corballis, 1988; Harris \& Dux, 2005). On the other hand, stored information about the usual orientation of the object can influence certain aspects of performance, such as the ease with which one can determine the object's spatial orientation and, as a result, one's ability to form distinct episodic representations of the object when it is repeatedly encountered.

\section{REFERENCES}

Arnell, K. M., \& Jolickur, P. (1997). Repetition blindness for pseudoobject pictures. Journal of Experimental Psychology: Human Perception \& Performance, 23, 999-1013.

Bavelier, D. (1994). Repetition blindness between visually different items: The case of pictures and words. Cognition, 51, 199-236.

Corballis, M. C. (1988). Recognition of disoriented shapes. Psychological Review, 95, 115-123.

DAVIDOFF, J., \& WARRINGTON, E. K. (1999). The bare bones of object recognition: Implications from a case of object recognition impairment. Neuropsychologia, 37, 279-292.

De Caro, S. A. (1998). On the perception of objects and their orientations. Spatial Vision, 11, 385-399.

De Caro, S. A., \& Reeves, A. (2000). Rotating objects to determine orientation, not identity: Evidence from a backward-masking/dual-task procedure. Perception \& Psychophysics, 62, 1356-1366.

Forster, K. I., \& Forster, J. C. (2003). DMDX: A Windows display program with millisecond accuracy. Behavior Research Methods, Instruments, \& Computers, 35, 116-124.

Harris, I. M., \& DuX, P. E. (2005). Orientation-invariant object recognition: Evidence from repetition blindness. Cognition, 95, 73-93.

HARRIS, I. M., HARRIS, J. A., \& CAINE, D. (2001). Object orientation agnosia: A failure to find the axis? Journal of Cognitive Neuroscience, 13, 800-812.

JolicEeUR, P. (1985). The time to name disoriented natural objects. Memory \& Cognition, 13, 289-303.

Kahneman, D., \& Treisman, A. M. (1984). Changing views of attention and automaticity. In R. Parasuraman \& D. R. Davies (Eds.), Varieties of attention (pp. 29-61). Orlando, FL: Academic Press.

KANWISHER, N. G. (1987). Repetition blindness: Type recognition without token individuation. Cognition, 27, 117-143.

Kanwisher, N. G., Yin, C., \& WoJCIUlik, E. (1999). Repetition blindness for pictures: Evidence for the rapid computation of abstract visual descriptions. In V. Coltheart (Ed.), Fleeting memories: Cognition of brief visual stimuli (pp. 119-150). Cambridge, MA: MIT Press.

Karnath, H.-O., Ferber, S., \& BülthofF, H. H. (2000). Neuronal representation of object orientation. Neuropsychologia, 38, 1235-1241.

Large, M.-E., McMullen, P. A., \& Hamm, J. P. (2003). The role of axes of elongation and symmetry in rotated object naming. Perception \& Psychophysics, 65, 1-19.

LEEK, E. C. (1998). Effects of stimulus orientation on the identification of common polyoriented objects. Psychonomic Bulletin \& Review, 5, 650-658.

LofTus, G. R., \& MAsson, M. E. J. (1994). Using confidence intervals in within-subject designs. Psychonomic Bulletin \& Review, 1, 476-490.

Milner, A. D., \& Goodale, M. A. (1995). The visual brain in action. Oxford: Oxford University Press.

SnOdgrass, J. G., \& VANDERWART, M. (1980). A standardized set of 260 pictures: Norms for name agreement, image agreement, familiarity, and visual complexity. Journal of Experimental Psychology: Human Learning \& Memory, 6, 174-215.

Turnbull, O. H., Beschin, N., \& Della Sala, S. (1997). Agnosia for object orientation: Implications for theories of object recognition. Neuropsychologia, 35, 153-163.

Turnbull, O. H., Della Sala, S., \& Beschin, N. (2002). Agnosia for object orientation: Naming and mental rotation evidence. Neurocase, 8, 296-305.

Turnbull, O. H., Laws, K. R., \& McCarthy, R. A. (1995). Object recognition without knowledge of object orientation. Cortex, 31, 387-395.

(Manuscript received June 17, 2004; revision accepted for publication November 29, 2004.) 